\title{
Warming Climate Shortens Ice Durations and Alters Freeze and Breakup Patterns in Swedish Water Bodies
}

Sofia Hallerbäck ${ }^{1,2}$, Laurie S. Huning ${ }^{3,1}$, Charlotte Love ${ }^{1}$, Magnus Persson ${ }^{2}$, Katarina Stensen ${ }^{4}$, David Gustafsson ${ }^{4}$, Amir AghaKouchak ${ }^{1,5}$

${ }^{1}$ Department of Civil and Environmental Engineering, University of California, Irvine, USA

2 Lund University, Sweden

3 Department of Civil Engineering and Construction Engineering Management, California State University, Long Beach, USA

${ }^{4}$ Swedish Meteorological and Hydrological Institute, Sweden

5 Department of Earth System Science, University of California, Irvine, USA 
For a review of previous studies on change in freeze, breakup date and ice duration, the reader is referred to Table $\mathrm{S} 1$. The studies represent different parts of the northern hemisphere and over different time periods. In Figure S2, we present the linear best fit of change per decade in ice duration, freeze and breakup date during 1913-2014 and 19592014. The figure shows a more substantial shift in the latter time period and more in the southern latitudes than the northern. In Figure S3 we present the correlations between ice variables (freeze, breakup and ice duration) and mean temperature for different time periods.

Table S1. Change in freeze date, breakup date, and ice duration (in days per decade, unless otherwise specified) for inland lakes and rivers, for multiple locations and time periods.

\begin{tabular}{|c|c|c|c|c|c|c|}
\hline \multirow[b]{2}{*}{ Reference } & \multirow[b]{2}{*}{$\begin{array}{l}\text { Location (Number and } \\
\text { Type of Water Bodies) }\end{array}$} & \multicolumn{2}{|c|}{ Study Period } & \multicolumn{3}{|c|}{ Change in } \\
\hline & & $\begin{array}{l}\text { Initial } \\
\text { Year }\end{array}$ & $\begin{array}{l}\text { Final } \\
\text { Year }\end{array}$ & $\begin{array}{l}\text { Freeze } \\
\text { Date }\end{array}$ & $\begin{array}{l}\text { Breakup } \\
\text { Date }\end{array}$ & $\begin{array}{c}\text { Ice } \\
\text { duration }\end{array}$ \\
\hline $\begin{array}{l}\text { Magnuson et al. } \\
(2000)\end{array}$ & $\begin{array}{l}\text { Northern Hemisphere ( } 39 \\
\text { lakes) }\end{array}$ & 1846 & 1995 & 0.58 & -0.65 & \\
\hline $\begin{array}{l}\text { Benson et al. } \\
(2012)\end{array}$ & $\begin{array}{l}\text { Northern Hemisphere ( } 9 \\
\text { lakes freeze, } 17 \text { breakup) }\end{array}$ & 1855 & 2005 & 1.1 & -0.89 & \\
\hline Takács (2011) & Danube river (1 river) & 1875 & 2004 & $\begin{array}{l}0.56 \text { to } \\
1.12\end{array}$ & $\begin{array}{l}-0.56 \text { to }- \\
0.96\end{array}$ & -0.56 to -2 \\
\hline $\begin{array}{l}\text { Latifovic and } \\
\text { Pouliot (2007) }\end{array}$ & Canada (20 lakes) & 1950 & 2004 & 1.2 & -1.8 & -3 \\
\hline $\begin{array}{l}\text { Jensen et al. } \\
(2007)\end{array}$ & $\begin{array}{l}\text { Great Lakes region (65 } \\
\text { lakes and rivers) }\end{array}$ & 1975 & 2004 & 3.3 & -2.1 & -5.3 \\
\hline $\begin{array}{l}\text { Latifovic and } \\
\text { Pouliot (2007) }\end{array}$ & $\begin{array}{l}\text { Canada (6 lakes in } \\
\text { northern Canada) }\end{array}$ & 1985 & 2004 & 7.6 & -9.9 & -17.5 \\
\hline \multirow[t]{2}{*}{ Hodgkins (2013) } & $\begin{array}{l}\text { US, New England (up to } 28 \\
\text { lakes) }\end{array}$ & 1908 & 2008 & & -0.4 & \\
\hline & $\begin{array}{l}\text { US, New England (up to } 28 \\
\text { lakes) }\end{array}$ & 1958 & 2008 & & -1.8 & \\
\hline Wang et al. (2012) & $\begin{array}{l}\text { Great Lakes region (over } \\
\text { all) }\end{array}$ & 1973 & 2010 & & & $-73 \%$ \\
\hline Surdu et al. (2014) & North Slope of Alaska & 1950 & 2011 & 5.9 & $\begin{array}{l}-17.7 \text { to } \\
18.6\end{array}$ & -24 \\
\hline $\begin{array}{l}\text { Patterson and } \\
\text { Swindles (2015) }\end{array}$ & Eastern North America & 1836 & 2013 & & Earlier & \\
\hline \multirow[t]{6}{*}{ This study } & Sweden (58 lakes) & 1914 & 2014 & 0.9 & -0.9 & -1.9 \\
\hline & - North of $60^{\circ} \mathrm{N}$ & & & 0.8 & -0.8 & -1.7 \\
\hline & - South of $60^{\circ} \mathrm{N}$ & & & 1.3 & -1.3 & -3.2 \\
\hline & Sweden (58 lakes) & 1959 & 2014 & 2 & -2.9 & -4.9 \\
\hline & - North of $60^{\circ} \mathrm{N}$ & & & 1.3 & -2.4 & -3.6 \\
\hline & - South of $60^{\circ} \mathrm{N}$ & & & 3.6 & -4.1 & -7.8 \\
\hline
\end{tabular}




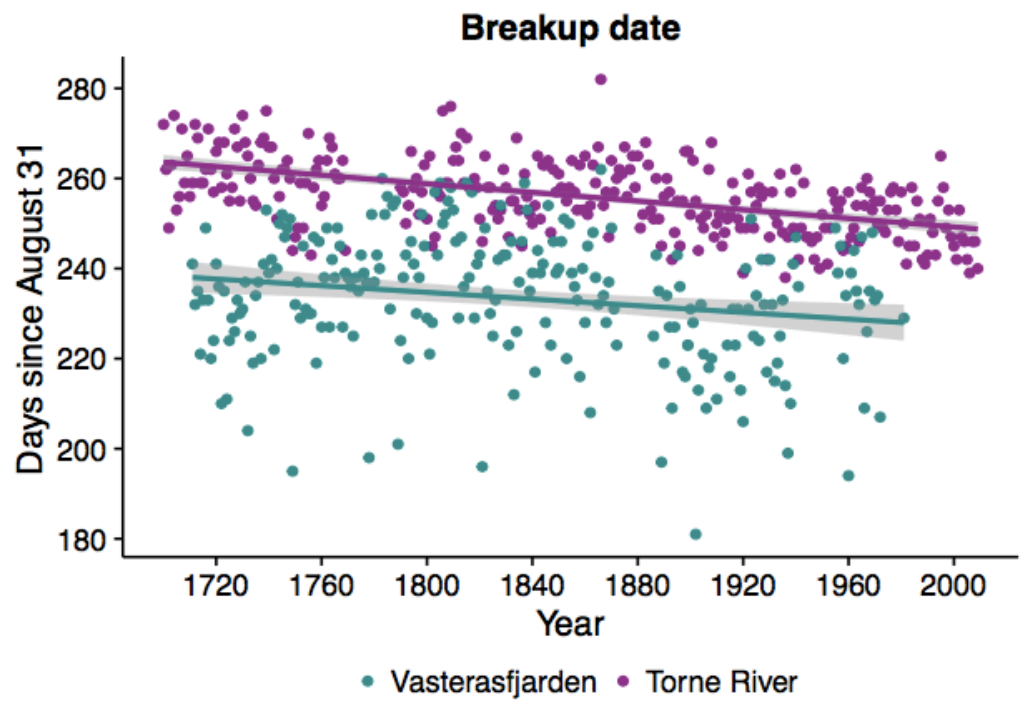

Figure S1. Observed breakup dates form 1700 - 2009 for Torne River and 1711 - 1989 for Västeråsfjärden. The best linear fit and the corresponding confidence levels are shown for both series. 


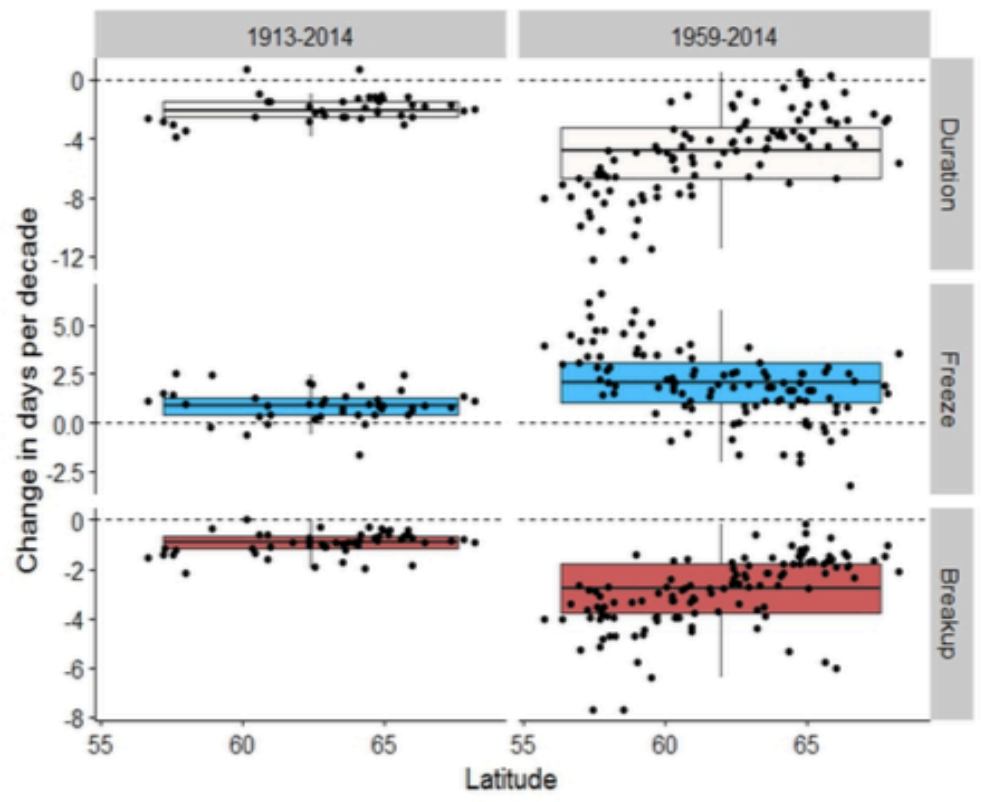

Figure S2. Change per decade in ice duration, freeze and breakup date during1913-2014 and 1959-2014. Decrease in freeze and breakup date represent earlier date, while increase represent a later date. Values for individual lakes are marked in black based on latitude. 


$\begin{array}{llll}\text { Temp mean, months } & \text { Ice duration } & \text { Freeze date } & \text { Breakup date } \\ \text { October-December, OND } & -0.44 & 0.50 & -0.11 \\ \text { December-February, DJF } & -0.34 & 0.19 & -0.35 \\ \text { March-May, MAM } & -0.44 & 0.07 & -0.69 \\ \text { April-June, AMJ } & -0.38 & 0.10 & -0.56 \\ \text { November-May, N-M } & -0.50 & 0.26 & -0.53 \\ \text { September-August, Annual } & -0.54 & 0.31 & -0.53\end{array}$

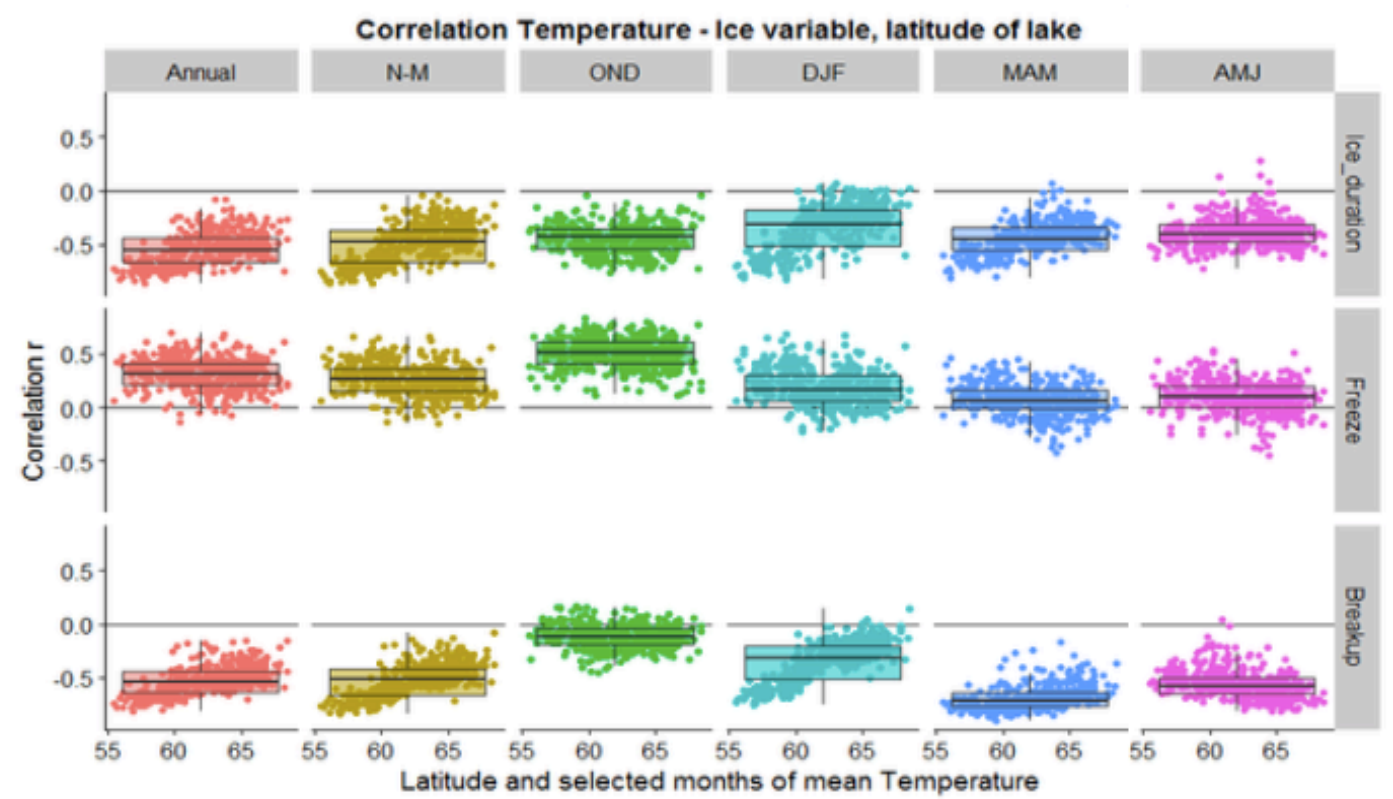

Figure S3. Correlations between ice variables (freeze, breakup and ice duration) and mean temperature for different time periods. Mean values are tabulated over different times of the year. Correlations during these same time periods are displayed across latitudes in the figure. 